\title{
Gobierno indígena y política social. Programa de Desarrollo Integral para la Tribu Yaqui, Sonora, México
}

\author{
Indian government and social policy. Integral \\ Development Program for Yaqui Tribe, \\ Sonora, Mexico
}

Recibido: 10 de diciembre de 2010

Francisca de la Maza ${ }^{1}$

Aprobado: 15 de marzo de 2011

\section{RESUMEN}

El tema general que se aborda es la situación contemporánea de los yaquis, pueblo indígena del noroeste de México, respecto a sus formas de gobierno indígena y la política social desarrollada por el gobierno federal y estatal mexicano entre los años 1989 y 2003. Durante este periodo se implementó el Plan Integral de Desarrollo para la Tribu Yaqui basado en la autogestión de los yaquis con importantes apoyos económicos tanto del gobierno federal como estatal. A partir de esto, se analiza algunos elementos descriptivos del aspecto organizacional indígena y de la política social mexicana desde una perspectiva teórica que da cuenta de diversas situaciones. Particularmente de la práctica política yaqui bajo el marco de la etnicidad, es decir, en la construcción de esta práctica en el tiempo por medio de la relación con el Estado mexicano y otros agentes externos bajo un sistema de dominación complejo, incluyendo ámbitos político, económico, social y cultural, que configuran un escenario actual de mutua influencia y dependencia.

Palabras clave: Estado-nación, política social, etnicidad, gobierno indígena.

\begin{abstract}
The general theme addressed is the contemporary situation of the Yaqui Tribe in northwestern Mexico, for their own indigenous forms of government and social policy developed by the Mexican state and federal governments between 1989 and 2003. During this period we implemented the Comprehensive Development Plan of the Yaqui tribe based on self-important of the Yaquis with financial support from both the federal and state government. From this, we analyze some descriptive elements of indigenous organizational aspect of Mexican social policy from a theoretical perspective that accounts for different situations. Particularly Yaqui political practice within the framework of ethnicity, namely the construction of this practice over time through the relationship with the Mexican government and other external agents under a complex system of domination, including political, economic, social and cultural, that make up a current scenario of mutual infuence and dependence.
\end{abstract}

Key words: Nation state, social policy, ethnicity, indigenous government. 


\section{Introducción}

El artículo se compone de tres partes: antecedentes generales que permiten situar a los yaquis desde una perspectiva histórica, un análisis de la política social contemporánea y las implicancias de esta en el sistema político Yaqui, y conclusiones. A partir de elementos descriptivos se analiza una perspectiva teórica que permite ahondar en las implicancias de las políticas sociales en los sistemas de gobierno indígena dentro de un contexto local y global $^{2}$.

Los yaquis habitan en el suroeste del estado de Sonora, México, comprendiendo aproximadamente 32 mil personas ${ }^{3}$. A pesar de que es un grupo no tan numeroso, es uno de los más representativos del estado de Sonora debido a sus características históricas y culturales.
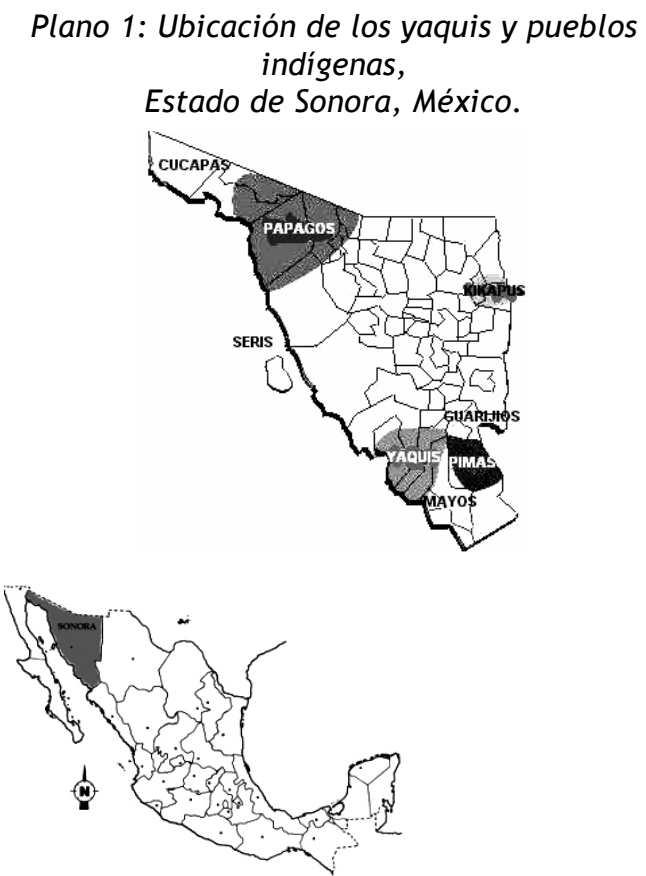

Fuente: Gobierno del estado de Sonora.
Uno de los aspectos más relevantes respecto a la historia de los yaquis es el reconocimiento que hizo en 1940 el Presidente de la República Lázaro Cárdenas dentro de su política agrarista. A través de un decreto reconoce a los yaquis un territorio continuo de 485.235 ha. Este hito se fundamenta en las luchas y demandas históricas de los yaquis por defender su territorio frente a diversos intentos de ocupación y despojo que incluyó reiteradas guerras, deportación y ocupación militar.

Tradicionalmente los yaquis habitan en ocho pueblos creados por la influencia jesuita a principios del siglo XVII, que perduran hasta el día de hoy, sin embargo, actualmente los pueblos no son exclusivos yaquis debido a que hay una alta convivencia con familias no yaquis, al igual que grupos importantes han migrado a las ciudades cercanas.

Actualmente el territorio Yaqui se compone de la Sierra del Bacatete, un área costera y un costado del Valle del Yaqui, este último caracterizado por su alta fertilidad y potencialidad agrícola. Los yaquis lograron conseguir un distrito de riego propio $^{4}$ y una concesión marítima de uso exclusivo.

Cada uno de los ocho pueblos mantiene un sistema político local compuesto por instancias civiles, militares y religiosas que son representadas por cinco autoridades: gobernador, pueblo mayor, capitán, comandante y secretario. Este sistema político rige en forma relativamente autónoma al interior de los pueblos, teniendo una influencia importante en los asuntos religiosos y políticos, pero que en forma creciente el gobierno Yaqui interactúa con los sistemas políticos del gobierno mexicano en sus tres niveles: municipal, estatal y federal.

A su vez, los ocho pueblos actúan como colectivo, autodefiniéndose como la "Tri-

2 Este artículo se basa en la investigación realizada para tesis de maestría durante el año 2003 dentro del Programa de Posgrado del Centro de Investigaciones y Estudios Superiores en Antropología Social. CIESAS, México. Se presentó una versión preliminar en al Congreso Chileno de Antropología de 2004.

3 Existe un grupo poblacional yaqui en el estado de Arizona, Estados Unidos, que se estableció luego de migraciones forzadas a principios del siglo XX.

4 Dentro del reconocimiento cardenista, también se incluyó el derecho al uso de un porcentaje de las aguas del río Yaqui, principalmente para irrigación agrícola, que posteriormente constituyó el Distrito de Riego de Comunidades Yaquis. Actualmente este distrito es el único que no se ha traspasado del Estado a los usuarios debido a los conflictos internos entre los yaquis. 
bu Yaqui", que idealmente reúne a las cinco autoridades de los ocho pueblos (en total 40 autoridades) en una asamblea general para resolver problemas que los afectan en conjunto, siendo el principal la ejecución del decreto cardenista, debido a que nunca se concretó la delimitación del territorio por problemas de interpretación del decreto y de los planos.

\section{Plano 2: Los ocho pueblos yaquis fundados por los jesuitas}

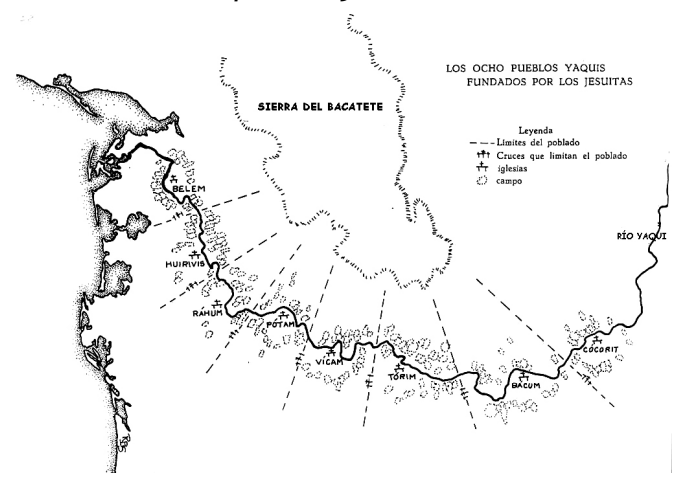

Fuente: Spicer (1994:32).

Otro aspecto importante de destacar para comprender la problemática que se discute es que los yaquis se han caracterizado históricamente como un grupo indígena que ha luchado por su territorio y por un sistema político-cultural propio y autónomo. La construcción de los yaquis como grupo étnico se ha caracterizado como "resistente", "persistente", "autónomo" y "guerrero" relacionado a las luchas por conservar su territorio -por las características geográficas y climáticas del Valle del Yaqui, muy fértil que permite el desarrollo agrícola en una zona semidesértica- y por su capacidad de resistir diversas políticas estatales como la deportación, la colonización y la ocupación militar.

Este estereotipo, imagen e idea sobre los yaquis, persiste en el imaginario colectivo de la sociedad mexicana, en parte en la sonorense y en particular de los propios yaquis. Esta idea, que la defino como primordialista y esencialista, se relaciona al concepto de la "invención de la sociedad primitiva" propuesto por Adam Kuper $(1988,2003)$ que sostiene que en la antropología ha primado la persistencia de un concepto ideal de "sociedad primitiva", homologado muchas veces con la "sociedad indígena".

El predominio de imágenes prototípicas ha impedido ver aspectos diferentes al interior de estas sociedades. La "ilusión" de esta idea, inventada por la antropología evolucionista, ha caracterizado el estudio de estas sociedades desde una perspectiva simplista. El prototipo se presenta en los estudios antropológicos y en la construcción de teoría. Este discurso, en el caso de los yaquis, se reproduce mítica y simbólicamente, estos luchan por mantener esa imagen, reproduciéndola en su discurso étnico, apoyado por estudios antropológicos que la sustentan ${ }^{5}$. Esta ilusión e invención de la sociedad Yaqui como esencialmente resistente y autónoma impide analizar su problemática desde una perspectiva de una sociedad compleja, tomando el concepto en el sentido que lo presenta Eric Wolf (1979), inserta en una trama de relaciones con la sociedad no Yaqui, donde se presenta constantemente una tensión entre la idea de los yaquis y su práctica concreta.

\section{Perspectiva teórica y metodológica}

La política social contemporánea se analiza desde una perspectiva que considera la etnicidad como un proceso que puede ayudar a dar cuenta de la interacción entre el Estado-nación y un grupo étnico, en este caso particular, entre el Estado mexicano y los yaquis. Se utiliza el concepto de la etnicidad ${ }^{6}$ desde una perspectiva constructivista ya que es suficientemente flexible y da cuenta del proceso histórico y relacional en que se construyen las diferencias, especialmente bajo un contexto de dominación, donde intervienen diversas variables: culturales, sociales, económicas y políticas. La visión constructivista

5 Los estudiosos de los yaquis destacan sus características organizacionales como autónomas y resistentes en diferentes periodos históricos, como Alfonso Fabila (1940), Edward Spicer (1994), Alejandro Figueroa (1994). A partir de estos estudios, probablemente, diversos autores nombran a los yaquis (al igual que al pueblo mapuche) como representativos de pueblos resistentes, como Guillermo de la Peña (1986) y Enrique Florescano (2001).

6 Los autores que aportan en esta concepción de etnicidad: Giménez, G. (2000), Pérez-Ruiz, M. (2002) y Vermeulen y Govers (1997). 
de la etnicidad permite analizar otros ámbitos más específicos como las identidades sociales, particularmente las identidades étnicas, y cómo intervienen diversos elementos en su construcción, especialmente a nivel de discursos como es la identidad desde una perspectiva primordialista e instrumentalista, lo cual no es excluyente, sino que ayuda a comprender el fenómeno desde una óptica más diversa y dinámica.

Dentro de esta perspectiva el Estado se considera una construcción que lleva detrás un proyecto político y que se manifiesta en lo local en forma fragmentaria y difusa, cobrando relevancia las agencias y los agentes y también los actores con los que interactúa. En este enfoque la política social se analiza como una manifestación particular de un proyecto ideológico, político y económico mayor que se concretiza en lo local, por medio de la acción de los agentes y de la relación directa con los miembros de la sociedad a quien van dirigidas ${ }^{7}$.

El tema de investigación que se presenta supone un análisis de procesos sociales y de sujetos interactuando, desde una perspectiva del presente y una mirada hacia el pasado. La etnografía como método permitió abordar estas temáticas a través de la perspectiva cualitativa, ya que dispone de una forma de recolectar e interpretar la información de forma flexible y comprensiva, incorporando diversas técnicas.

Se realizó una revisión bibliográfica y un análisis de documentos del Fondo Documental del Instituto Nacional Indigenista en Ciudad de México con el fin de conformar un contexto general que permitiera ahondar en las bases de la investigación.

El trabajo de campo comprendió cuatro meses en el pueblo de Pótam, pero también fue necesario abordar de forma general los otros pueblos yaquis, debido a que Pótam forma parte del "Sistema Político Yaqui Regional" que involucra a los 8 pueblos yaquis.

Metodológicamente, desde la llegada a Pótam, se intentó participar en las actividades políticas, las cuales contemplaban las asambleas rutinarias los días domingos y asambleas generales donde participaban otros pueblos, ya sea para discutir asuntos internos o recibir visitas externas. Fue fundamental para el seguimiento de la actividad política la asistencia a estas asambleas debido a que se estableció un contacto permanente con la actividad política y con sus participantes, principalmente las personas con cargos políticos. También se asistió a ceremonias religiosas del Día de Muertos, la Cuaresma y la Semana Santa, lo que dio una dimensión mayor a las actividades al interior de los pueblos yaquis.

Se realizó observación y participación directa en las actividades, también se contó con material de hemeroteca y diversos documentos estatales y federales y cartas o comunicados de las autoridades yaquis. Se realizaron formalmente 30 entrevistas directas a personas destacadas en el proceso político, junto con múltiples conversaciones informales tanto en la vida cotidiana como en la práctica política Yaqui y no Yaqui.

Uno de los aspectos importantes a señalar es que se decidió abordar la temática de estudio de una forma amplia que permitiera dar cuenta de un proceso extremadamente complejo, debido a que la profundidad en todos los aspectos no era posible de realizar en los meses de campo.

Respecto a la relación con los yaquis, con las autoridades, con los hombres y mujeres, con y sin cargos, fue muy favorable, teniendo siempre una buena acogida tanto de la familia que me recibió en su casa y de mucha gente que apoyó y prestó su testimonio de la década de estudio. La confianza en el desarrollo de la investigación de parte de líderes yaquis también fue fundamental para lograr sustentar las entrevistas con documentación generada por los propios yaquis y por entidades estatales y federales.

Durante el periodo de campo, una facción tenía un plantón en la carretera internacional que llevaba 10 meses, unido a esto, el estado de Sonora se encontraba en un periodo electoral, lo cual marcó la estancia con una

\footnotetext{
Para este análisis de toman los siguientes autores: Abrams, P. (1977), Corrigan y Sayer (1985) y Gupta, A. (1988).
} 
fuerte efervescencia política. Otro aspecto que dificultó el trabajo es la situación de riesgo en el pueblo de Pótam, principalmente debido a la pobreza y falta de trabajo. También fueron dificultades el uso de la lengua Yaqui tanto en las asambleas y reuniones como en lo cotidiano, y la participación en un espacio político, principalmente masculino.

\section{Resultados y discusión}

En México, a principios de los 80 y coincidente con el cambio presidencial se cuestiona la política indigenista (política social hacia indígenas) desde el organismo que debe aplicarla: el Instituto Nacional Indigenista (INI). Este intento de transformación está fuertemente influido por las corrientes vinculadas al etnodesarrollo expresadas en la "Declaración de San José sobre el Etnocidio y el Etnodesarrollo" (Bonfil et al. 1982). Como idea de implementar una nueva forma de desarrollo, el INI sugiere realizar una experiencia de etnodesarrollo inicial con los yaquis, debido entre otras cosas a que habían tenido problemas con la oficina local del INI.

Esta experiencia se llamó "Plan de Desarrollo Integral para la Tribu Yaqui" (PIDTY) que consistió en una primera etapa en un diagnóstico participativo y luego se desarrollaría un plan de desarrollo con actividades específicas. Estas últimas, por diversas circunstancias, no se llevaron a cabo, solo hasta su reformulación dentro del sexenio presidencial de Carlos Salinas de Gortari, coincidente con las reformas económicas neoliberales impulsadas por su gobierno. El Plan tuvo una duración de 10 años (1989-1999), fue formulado y ejecutado por líderes y profesionales yaquis y avalado por las autoridades tradicionales de la Tribu Yaqui.

En términos generales el Plan consistía en abarcar el desarrollo en forma integral en diferentes áreas: económica, social, educación, salud, cada una con su plan de acción, en el cual tienen un rol protagónico jóvenes profesionales yaquis (agrónomos) y maestros bilingües. Sus objetivos eran: reactivar las principales ramas de producción (agrícola, pesca y ganadería), diversificar el uso de recursos naturales disponibles, incrementar los índices de bienestar social y contribuir al fortalecimiento de la identidad cultural.
El plan tenía grandes pretensiones como: lograr autosuficiencia alimentaria, disponer de excedentes para su comercialización, ampliar las oportunidades de empleo, recuperar la capacidad de compra de las familias, satisfacer las necesidades de servicio de agua potable, electrificación, drenaje, vivienda, educación, salud y caminos.

Para ejecutar el Plan se creó un sistema para traspasar los recursos directamente a los profesionales yaquis desde el gobierno federal y estatal, apoyados por PRONASOL (Programa Nacional de Solidaridad), sin pasar por instituciones intermedias debido a que el sistema político Yaqui y sus autoridades no cuentan con un reconocimiento legal de parte del Estado mexicano. Se creó un fideicomiso (o fondo económico) apoyado por una estructura organizacional compuesta mayoritariamente por representantes y profesionales yaquis, los cuales tuvieron a cargo disponer los recursos y fueron responsables de su aplicación por medio de proyectos concretos.

El Plan contó con un equipo técnico compuesto principalmente por jóvenes yaquis que ejecutan los diversos programas, superando las 60 personas, los cuales fueron conocidos como los "paticys". PATICY es la sigla del "Programa de Asistencia Técnica Integral de Comunidades Yaquis" que corresponde a la parte visible y técnica del programa que se aplicaba directamente en los pueblos yaquis.

El Plan de Desarrollo Integral fue una experiencia única en México en términos de su duración, los recursos económicos dispuestos, su difusión a nivel nacional, la disposición federal y estatal para su implementación y la ejecución y manejo de recursos por los propios beneficiarios indígenas. Sin embargo, se puede decir que no tuvo los alcances esperados, y aún más, se agudizaron conflictos internos entre autoridades yaquis, profesionales yaquis y yaquis en general. El desarrollo del Plan estuvo marcado por alianzas políticas, particularmente vinculadas al partido de gobierno federal y estatal, el Partido Revolucionario Institucional-PRI.

Este Plan tuvo diversos impactos en los pueblos yaquis y en el gobierno indígena. Durante una década se canalizaron fuertes sumas de dinero, pero sus impactos económicos 
fueron pocos: los yaquis se convirtieron en rentistas de sus tierras, de las $25 \mathrm{mil}$ ha irrigadas con que cuenta su territorio, sobre el $90 \%$ están rentadas a personas externas (principalmente empresarios agrícolas), la infraestructura de los pueblos mejoró en algunos sentidos pero en general no cuentan con servicios básicos como alcantarillado, agua potable y alumbrado público, las fuentes de trabajo tuvieron que buscarse fuera de los campos, hoy un grupo importante de jóvenes trabajan en maquilas y campos agroindustriales.

En términos sociales, se agudizaron diferencias socioeconómicas al interior de los yaquis, entre los que tenían su fuente de trabajo segura -que participaban como ejecutores del Plan y sus allegados más cercanos que se beneficiaron más directamente de los programas- y el resto de la población. Los profesionales, asesores internos y externos, adquirieron un poder creciente sobre las autoridades tradicionales, también vinculándose a organizaciones indígenas nacionales y partidos políticos. Las demandas de apoyo en dinero de los grupos dirigentes hacia el gobierno federal y estatal, aumentaron. Los malos manejos de dinero también fueron evidentes.

Respecto al impacto en el gobierno Yaqui, se cristalizó una división interna el año 1994, creando duplicidad de autoridades en tres pueblos yaquis. Esta división se conforma a partir de la disidencia al Plan de las autoridades de estos tres pueblos. Esto ocasionó diversos enfrentamientos al interior de los pueblos yaquis, incluyendo muertos, heridos, incendio de casas, toma de lugares y oficinas y cierre de calles.

El desarrollo del Plan y las múltiples demandas de los líderes yaquis ligadas especialmente a la ratificación del decreto cardenista de reconocimiento del territorio Yaqui lleva a que en el año 1997 se firme un convenio con el Presidente de la República Ernesto Zedillo, de restitución del territorio Yaqui con una superficie de $459 \mathrm{mil} \mathrm{ha}$. Este convenio implicaba la expropiación de un sector del territorio de $2688 \mathrm{ha}$, por lo cual los yaquis recibirían una suma de dinero conformando el FIFONAFE (Fideicomiso Fondo Nacional de Fomento Ejidal).
Sin embargo, hasta el años 2003 no se había ejecutado ni utilizado el fideicomiso por las mismas razones de diferencias de interpretación del decreto cardenista y agudizado por los conflictos internos

En 1999, debido a estos conflictos, se da por terminado el Plan de Desarrollo Integral, cerrándose el fondo económico que los respaldaba. En los años siguientes se conforman dos alianzas políticas dentro del sistema político Yaqui: los llamados "paticys" y los del Bahugo. Los primeros se componen de un grupo de profesionales yaquis, vinculados al Plan, y de autoridades tradicionales. Estos logran seguir trabajando a través de una nueva instancia estatal llamada CEPRODE (Centro de Promoción de Desarrollo), dependiente del gobierno del estado de Sonora. Mientras, el segundo grupo también se integra por un grupo de profesionales yaquis y autoridades, ambos disidentes del Plan. Estos mantienen en el año 2003 un plantón permanente en la carretera internacional (México-EE.UU.) que cruza el territorio Yaqui en el sitio llamado el Bahugo. Ambas alianzas tienen demandas similares, los dos grupos negocian y ejecutan diversas medidas de presión con las diferentes instancias federales y estatales (ver anexo).

En general, el gobierno Yaqui se caracteriza por la conformación de una clase política diferenciada de la comunidad política: los miembros de los pueblos yaquis ${ }^{8}$. Esta clase política está dedicada exclusivamente a la actividad política conformada por las autoridades tradicionales yaquis y sus seguidores más cercanos, hombres dedicados a acompañar y a apoyar a las autoridades y con funciones de vigilancia, y un grupo de "mujeres de apoyo", mientras el resto de la comunidad se mantiene marginal y solo participa excepcionalmente en las actividades políticas.

La clase política Yaqui, liderada por jóvenes profesionales, hace un uso instrumental y primordialista de la imagen Yaqui en sus formas de negociación, distante de la comunidad política Yaqui e incapaz de ser efectiva en una sociedad con sus características actuales. El gobierno Yaqui cumple un rol más simbólico y ritual, muy importante en la vida Yaqui, pero ya incapaz de abordar otros ámbitos como los

Se utilizan conceptos de antropología política para analizar el sistema político basándose principalmente en Easton (1988) y Bailey $(1972,1980)$. 
políticos, sociales y económicos que afectan a la comunidad en su conjunto.

Los conflictos internos actuales crean condiciones para que esta clase política negocie con niveles del Estado e instancias externas sin tener una real comunicación con la gente común de los pueblos. Las decisiones políticas, e incluso el conflicto político, son de la clase política, mientras las familias logran adecuarse a estas diferencias y hacer un uso instrumental de ellas. Estos conflictos tienen diversas explicaciones, por un lado, originados por la propia estructura interna de la sociedad Yaqui, agudizada por su crecimiento y complejidad, y por otro lado, un faccionalismo que se ha manifestado en momentos de crisis, donde los profesionales yaquis, sus líderes, han tenido una participación importante en este, unido también a los impactos de las políticas estatales.

La relación con agentes externos, particularmente a través de la implementación de la política social, se ha vuelto una forma de negociación interminable donde la amenaza Yaqui siempre está presente. Se convierte en un juego político entre las clases políticas, excluyendo a la población. Las demandas se incrementan pero también se mantienen en el tiempo, pasan a ser una forma de conseguir recursos, mientras las instancias estatales tienen un papel importante con formas de control, apoyadas por lealtades con líderes yaquis y por utilizar la división como una forma de condicionar cualquier tipo de negociación. Con esta situación, los resultados de las demandas y de las presiones de los yaquis son solo en un nivel intermedio y a corto plazo.

La clase política Yaqui tiene un patrón de comportamiento político que se caracteriza en negociar sin intermediarios, directamente con el Presidente de la República o el gobernador del estado a través de visitas y cartas, si sus demandas no son escuchadas en tiempos razonables, realizan medidas de presión como la toma de carreteras, detener el tránsito internacional, cierre de oficinas estatales y federales y de las llaves de un acueducto que pasa al interior de su territorio. Estas medidas son altamente efectivas, donde se conjugan diversos aspectos como la imagen y amenaza Yaqui, el pueblo resistente guerrero con alta confrontación, utilizada por los yaquis en for- ma instrumentalista en la clase política y también permeada en la sociedad y las instancias gubernamentales.

En el discurso de los líderes yaquis, el gobierno estatal de origen priista se considera como uno de los principales causantes de los conflictos y división interna, como un mecanismo de control político. Esta afirmación tiene que matizarse debido a que los líderes yaquis también utilizan esta relación para adquirir más poder político y económico y formas de control interno, estableciendo alianzas con diversos partidos políticos.

Las explicaciones del fracaso del Plan de Desarrollo Integral pueden ser diversas, pero lo relevante de destacar es que la sociedad Yaqui, su sistema político, se vio atravesado por diferentes aspectos como afiliaciones partidarias, intereses económicos, mal manejo de recursos financieros, estratificaciones socioeconómicas, debilidades en el sistema político, identidades étnicas y sociales flexibles y discursos étnicos esencialistas.

\section{Conclusión}

El breve recorrido respecto a las incidencias de la política social entre 1989 y 2003 en el territorio Yaqui permite señalar algunas conclusiones que dan cuenta de los impactos de la relación entre lo local y lo global, entre el gobierno indígena Yaqui y el sistema de gobierno mexicano.

Se puede decir que la práctica política yaqui actual, idealmente definida como autónoma y de confrontación con los agentes externos, tiene muchas más facetas y relaciones con aspectos que se tienden a considerar como "no yaquis". Esto significa, que el límite entre lo "tradicional" -lo propiamente Yaquicon lo no Yaqui es sumamente flexible, difuso y variable y depende de los contextos específicos. El análisis de la política social desarrollada durante estos años y su impacto al interior de los pueblos yaquis, específicamente en el gobierno Yaqui, da cuenta con claridad de las múltiples relaciones, interacciones entre los yaquis y los no yaquis y entre diversos sistemas políticos y económicos que interactúan en la región Yaqui. 
Sobre la utilidad del concepto de etnicidad se vio como un elemento importante al describir la construcción de las fronteras étnicas entre yaquis y el resto de la sociedad. Las evidencias mostraron que estas fronteras, a pesar de estar plagadas de un imaginario como fuertemente constituidas, son mucho más flexibles debido a la alta convivencia y a las dificultades de definir patrones genealógicos (tradicionales) de pertenencia, siendo finalmente el criterio de las autoridades tradicionales quienes definen la membresía al grupo. A pesar de esto, el mito de pueblo cerrado perdura como una fuente de construcción de identidad, principalmente en relación al otro no Yaqui y en las negociaciones con agentes externos.

La realidad presente en los pueblos yaquis muestra una sociedad compleja e inserta en la sociedad regional sonorense, interactuando en diversos niveles, lo cual en el análisis es necesario incorporar otros aspectos como las diferenciaciones en clases sociales al interior de los pueblos y formas de hacer política inserta en las tramas regionales y nacionales.

El poder y control político del gobierno Yaqui es restringido, opera en ciertos niveles, mientras otros poderes más estructurales tienen mayor influencia. Estos poderes estructu- rales se manifiestan en las relaciones que no solo operan dentro de escenarios y campos, sino que también las organizan y dirigen como lo señala Wolf (2001), constituyendo los poderes económicos y políticos globales, que van más allá del territorio Yaqui, del estado de Sonora y de México.

Se puede concluir que la política social como proyecto social y económico originado dentro del marco de los Estados Nacionales -Estado mexicano-, no se sustrae de políticas globales, específicamente económicas y políticas, que a corto y largo plazo tienen efectos directos a nivel local, donde no tiene importancia si son o no premeditadas o predecibles, pero que en concreto se insertan violentamente en un quehacer local con impactos profundos en las estructuras sociales, políticas y económicas de los grupos sociales. En particular, en relación a las poblaciones indígenas, estas suelen ser de mayor envergadura debido a que los procesos de desintegración forzada, desde el Estado, producto de persecuciones políticas, presión sobre la tierra y recursos naturales y sus sistemas culturales, son de mayor alcance. El Estado-nación como proyecto ideológico, plasmado en grupos económicos y políticos concretos, con alcances globales, agudiza la relación de dominación y se manifiesta con niveles dramáticos y probablemente irreversibles en las poblaciones indígenas. 
Anexo: División del sistema político (1989-2003)

\begin{tabular}{|c|c|c|}
\hline & 5 Pueblos: Los Paticys & 6 Pueblos: Movimiento el Bahugo \\
\hline Pueblos 9 & $\begin{array}{l}\text { Loma de Guamúchil (Cócorit) } \\
\text { Loma de Bácum (Bácum) } \\
\text { Vícam Estación (Vícam) } \\
\text { Pótam Pinos (Pótam) } \\
\text { Belem Tradicional (Belem) }\end{array}$ & $\begin{array}{l}\text { Tórim } \\
\text { Vícam Pueblo o Tradicional (Vícam) } \\
\text { Pótam Tradicional (Pótam) } \\
\text { Ráhum } \\
\text { Huírivis } \\
\text { Belem Dual (Belem) }\end{array}$ \\
\hline $\begin{array}{l}\text { Origen y año de } \\
\text { consolidación }\end{array}$ & $\begin{array}{l}\text { Origen: } 1989 \text { con PIDTY y equipo } \\
\text { de Paticy } \\
\text { Consolidación: } 2000 \text { por Ceprode }\end{array}$ & $\begin{array}{l}\text { Origen: } 1994 \text { disidencia del PIDTY de } \\
\text { Vícam y Pótam } \\
\text { Consolidación: } 2001 \text { por movimiento } \\
\text { del Bahugo }\end{array}$ \\
\hline Demandas & $\begin{array}{l}\text { Ejecutar el lindero cardenista - vía } \\
\text { Convenio Zedillo } \\
\text { Uso del dinero de FIFONAFE } \\
\text { Traspaso del Distrito de Riego } \\
\text { Desarrollo por pueblo - vía } \\
\text { CEPRODES } \\
\text { Autogestión de programas por } \\
\text { profesionales yaquis }\end{array}$ & $\begin{array}{l}\text { Ejecutar lindero cardenista - } \\
\text { vía revisión Convenio Zedillo: } \\
\text { cuestionamiento de } 40 \text { mil ha } \\
\text { Congelar uso de dinero FIFONAFE } \\
\text { hasta aclarar asunto de lindero } \\
\text { Traspaso del Distrito de Riego } \\
\text { Desarrollo Estratégico e Integral en la } \\
\text { Tribu Yaqui } \\
\text { Autogestión de programas por } \\
\text { profesionales yaquis }\end{array}$ \\
\hline Tipo de acciones & $\begin{array}{l}\text { Negociación con agentes federales } \\
\text { y estatales } \\
\text { Cuenta a nivel municipal con } \\
\text { regidor étnico y un comisario } \\
\text { Acción vía CEPRODE, profesionistas } \\
\text { con sueldo estable } \\
\text { Toma de oficina de Comisión } \\
\text { Nacional del Agua } \\
\text { Negociación con candidatos } \\
\text { priistas (Partido Revolucionario } \\
\text { Institucional) }\end{array}$ & $\begin{array}{l}\text { Negociación con agentes estatales y } \\
\text { federales } \\
\text { Cuenta a nivel municipal con } \\
\text { coordinador de asuntos indígenas y un } \\
\text { comisario } \\
\text { Plantón permanente y solicitud de } \\
\text { dinero a automovilistas que apoya } \\
\text { financiamiento } \\
\text { Participación en organizaciones } \\
\text { indígenas nacionales: ANIPA } \\
\text { Negociación con candidatos } \\
\text { panistas(Partido de Acción Nacional) }\end{array}$ \\
\hline $\begin{array}{l}\text { Relación con } \\
\text { miembros de la } \\
\text { comunidad política }\end{array}$ & $\begin{array}{l}\text { Solicitud de becas escolares } \\
\text { Apoyo directo a familias y } \\
\text { actividades religiosas } \\
\text { Cartas para apoyo estatal en } \\
\text { diversas áreas } \\
\text { Participación de un sector de la } \\
\text { población en algunas actividades } \\
\text { políticas }\end{array}$ & $\begin{array}{l}\text { Solicitud de apoyo para proyectos de } \\
\text { Fondos Regionales del INI } \\
\text { Apoyo directo a familias y actividades } \\
\text { religiosas } \\
\text { Cartas de apoyo estatal en diversas } \\
\text { áreas } \\
\text { Participación de un sector de la } \\
\text { población en algunas actividades } \\
\text { políticas }\end{array}$ \\
\hline
\end{tabular}

$9 \quad$ El conflicto interno entre autoridades y líderes a favor o en contra del PIDTY ocasionó la duplicidad de autoridades en tres pueblos, lo cual creó alianzas entre las autoridades de los pueblos yaquis. Ninguna de las dos alianzas se compone de los 8 pueblos yaquis tradicionales. 


\section{Bibliografía}

ABRAMS, P. (1977), Notes on the difficulty of studying the State. En Journal of Historical Sociology, vol. 1, N¹, march 1988, pp. 58-89.

BAILEY, F.G. (1972), Decisions by Consensus in Councils and Committees: with special reference to village and local government in India. En Political systems and the distribution of power, M. Banton (ed.). A.S.A. Monographs 2, Tvistock Publications, USA, pp. 1-20.

BAILEY, F.G. (1980), Stratagems and spoils. A social anthropology of politics, Oxford Basil Blackwell, London.

BARNARD \& SPENCER (1996), Ethnicity. En Encyclopedia of social and cultural anthropology. Routledge, pp. 190-193.

BONFIL, G. et al. (1982), América Latina: etnodesarrollo y etnocidio. Ediciones FLACSO, Costa Rica.

CORRIGAN Y SAYER (1985), Introduction. En The Great Arch: English State Formation a cultural revolution. Oxford University Press, pp. 1-13.

DE LA MAZA, F. (2003), El sistema político yaqui contemporáneo: un análisis del gobierno, los conflictos y su relación con el Estado mexicano en el pueblo yaqui de Pótam, Sonora. Tesis que para obtener el grado de Maestra en Antropología Social, CIESAS, México.

DE LA PEÑA, G. (1986), La antropología sociocultural y el estudio del poder. En Poder y dominación. Perspectivas antropológicas, Villa Aguilera, M. (ed.) URSHSLAC / El Colegio de México, México, pp. 45-70.

EASTON, D. (1988), Esquema para el análisis político. Amorrortu Editores, Argentina.

FABILA, A. (1940), Las tribus yaquis de Sonora. Su cultura y anhelada autodeterminación. Primer Congreso Indigenista Interamericano, Departamento de Asuntos Indígenas, México.

FIGUEROA, A. (1994), Por la tierra y por los santos. Identidad y persistencia cultural entre yaquis y mayos. CONACULTA, México.

FLORESCANO, E. (2001). Etnia, Estado y Nación. Editorial Taurus, México.
GIMÉNEZ, G. (2000), Identidades étnicas: estado de la cuestión. En Los retos de la etnicidad en los estados-nación del siglo XXI, L. Reina (coord.). Ediciones CIESAS- INI, México pp. 45-70.

GUPTA, A. (1995). Blurred boundaries: the discourse of corruption, the cultura of politics, and the imagined state. En American Ethnologist, vol. 22 2:375-402.

KUPER, A. (1988). The invention of primitive society. Transformations of an ilussion. Routledge, London.

KUPER, A. (2003). The return of the native. En Current Anthropology, vol. 44 3::389-402.

PÉREZ RUIZ, M. (2002). El estudio de las relaciones interétnicas en la antropología mexicana. En Los estudios culturales en México, M. Valenzuela (coord.). Fondo de Cultura Económica, México, pp.116-207.

SPICER, E. (1994). Los yaquis. Historia de una cultura. UNAM, México.

VERMEULEN \& GOVERS edited (1997). The political of ethnic consciousness, St. Martin's Press, London.

WOLF, E. (1979). Aspectos de las relaciones de grupo en una sociedad compleja: México. En Campesinos y sociedades campesinas, T. Shanin, Fondo de Cultura Económica, México, pp. 43-95.

WOLF, E. (2001). Introducción y conceptos polémicos. En Figurar el poder. Ideologías de dominación y crisis, CIESAS, México pp. 15-95. 